\title{
Employer Branding vs. Internal Branding - Ein Vorschlag zur Integration im Rahmen der identitätsbasierten Markenführung
}
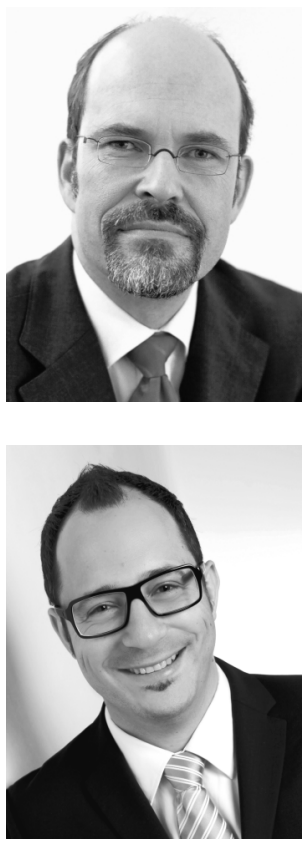

die entscheidend zum Unternehmenserfolg beitragen können. Daher ist es das Ziel des vorliegenden Artikels, die Konzepte Employer Branding und Internal Branding hinsichtlich Begriff, Zielsetzung und Zielgrössen sowie Instrumenten vorzustellen. Des Weiteren werden Gemeinsamkeiten und Unterschiede erörtert sowie ein Ansatz zur Integration im Rahmen der identitätsbasierten Markenführung erarbeitet.

With employer branding and internal branding two branding concepts gained attention of research and practice in the last decade. Both concepts focus on the target group of employees. While employer branding is concerned with positioning the organization as an attractive employer in order to attract potential employees and to retain current employees, internal branding focuses on current employees' fulfilment of the brand promise which was communicated to relevant external target groups. Although the objectives of employer branding and internal branding differ they constitute complementary concepts which contribute to organizational success. Therefore, the paper's objective is to present the concepts of employer branding and internal branding regarding definition, objectives and measures. Furthermore, similarities and differences will be discussed and an approach for integration within the framework of identity-based branding will be presented. 


\section{Warum Employer Branding vs. Internal Branding?}

Die Begriffe Employer Branding und Internal Branding sind in den letzten Jahren sowohl in der Unternehmenspraxis als Schlagworte für eine auf Mitarbeiter ausgerichtete Markenführung als auch in der Wissenschaft im Rahmen von Forschungsbemühungen stärker in den Fokus des Interesses gerückt. Dies ist einerseits darauf zurückzuführen, dass die Etablierung des Stakeholderbegriffs in der marktorientierten Unternehmensführung dazu geführt hat, dass Unternehmen neben klassischen Marktteilnehmern, wie Nachfragern und Wettbewerbern, auch andere Stakeholder, wie z.B. Mitarbeiter, im Rahmen ihrer Tätigkeit als Zielgruppe berücksichtigen (Ewing et al. 2002; Meffert et al. 2012). Die Relevanz der Mitarbeiter als Zielgruppe wird dabei aus ressourcenorientierten Ansätzen (Barney 1991) abgeleitet, nach denen Mitarbeiter eine Quelle für nachhaltige Wettbewerbsvorteile darstellen (Backhaus/Tikoo 2004; Moroko/Uncles 2008). Der Unternehmenserfolg kann demnach durch Investition in Mitarbeiter als Humankapital gesteigert werden (Backhaus/ Tikoo 2004).

So ergeben sich im Rahmen des Employer Branding Kostenvorteile aufgrund höherer Effizienz bei der Personalbeschaffung (Berthon et al. 2005; Barrow/Mosley 2006) und aufgrund geringerer Fluktuation (Backhaus/Tikoo 2004; Moroko/Uncles 2008; Martin et al. 2011; Kucherov/Zavyalova 2012; Elving et al. 2013) sowie Produktivitätsvorteile aufgrund höherer Leistung (Backhaus/Tikoo 2004; Forster et al. 2012; Kucherov/Zavyalova 2012). Tatsächlich weisen Fulmer et al. (2003) nach, dass Unternehmen, die Wert auf positive Beziehungen zu ihrem Mitarbeitern legen, nicht nur stabile und hochgradig positive Mitarbeitereinstellungen erzielen, sondern auch in Bezug auf marktbezogene und finanzielle Zielgrössen relative Vorteile gegenüber dem Markt aufweisen. Aufgrund dieser Vorteile versuchen Unternehmen, leistungsfähige Mitarbeiter zu gewinnen (Backhaus/Tikoo 2004; Barrow/Mosley 2006; Moroko/Uncles 2008), die zum Unternehmen passen (Mosley 2007; Sponheuer 2010; Forster et al. 2012), sowie diese Mitarbeiter zu binden und zu halten (Backhaus/Tikoo 2004; Kucherov/Zavyalova 2012; Elving et al. 2013). Um diese Ziele zu erreichen, müssen sich Unternehmen durch Employer Branding als attraktive Arbeitgeber profilieren (Ewing et al. 2002). Diese Profilierung gewinnt zusätzlich durch den Mangel an Fach- und Führungskräften an Bedeutung, der unter dem Schlagwort „war for talent“ Eingang in Wissenschaft und Praxis gefunden hat (Kirchgeorg/Günther 2006; Moroko/ Uncles 2008; Elving et al. 2013).

Schliesslich ist Employer Branding auch im Hinblick auf das Internal Branding von Relevanz. So wird im Rahmen einer markenorientierten Personalrekrutierung und -selektion bereits Markenwissen bei den Mitarbeitern aufgebaut (Piebler 2011). Des Weiteren stehen im Rahmen des Employer Branding bei Personalrekrutierung und Personalselektion nicht einfach die besten potentiellen Mitarbeiter im Fokus, sondern die besten zur Marke passenden potentiellen Mitarbeiter, d.h. Mitarbeiter mit einem hohen Mitarbeiter-Marke-Fit (Mosley 2007; Sponheuer 2010; Forster et al. 2012). So führt eine markenorientierte Personalrekrutierung zu einer Selbstselektion von potentiellen Mitarbeitern, da sich potentielle Mitarbeiter, die einen geringen Fit zwischen ihrer personalen Identität und der Markenidentität wahrnehmen, aufgrund von Selbstkonsistenzmotiven nicht bewerben werden (Judge/Cable 1997; Carless 2005; Chapman et al. 2005). Darüber hinaus kann eine markenorientierte Personalselektion einen hohen Fit zwischen Mitarbeitern und Marke sicherstellen (De Chernatony et al. 2003). Der zentrale Vorteil eines hohen Mitarbeiter-MarkeFit besteht für die markenführende Institution darin, dass der Sozialisationsaufwand gerin- 
ger ausfällt, wenn bereits ein hoher Mitarbeiter-Marke-Fit bei Eintritt vorliegt. Aufgrund von Erkenntnissen aus der Organisationsforschung (Dutton et al. 1994; Verquer et al. 2003; Arthur Jr. et al. 2006) und der Internal Branding-Forschung (Strödter 2008; Morhart et al. 2009) ist darüber hinaus anzunehmen, dass der Mitarbeiter-Marke-Fit zentrale Internal Branding-Zielgrössen wie Markenidentifikation, Brand Commitment und Brand Citizenship Behaviour beeinflusst.

Darüber hinaus kann eine Steigerung des Unternehmenswertes auch durch eine Steigerung des Kundenstammwertes und des Markenwertes als marktliche Vermögenswerte erreicht werden (Meffert et al. 2012). Zentrale Voraussetzung für die Steigerung von Marken- und Kundenstammwert ist insbesondere in stagnierenden, wettbewerbsintensiven Märkten die Kundenbindung (Homburg/Brubn 2010), die ein zentrales Ziel der Markenführung darstellt (Burmann/Meffert 2005; Burmann et al. 2005; Meffert et al. 2012). Grundlage langfristig stabiler Marke-Kunden-Beziehungen ist das der Marke entgegengebrachte Vertrauen (Delgado-Ballester et al. 2003; Sander/Weywara 2006; Wenske 2008). Als wesentliche Quellen des Vertrauens wiederum werden in der Literatur Zufriedenheit (Homburg et al. 2005; Huber et al. 2006; Homburg et al. 2010) und Glaubwürdigkeit (Ravald/Grönroos 1996; Eisend 2003; Zeplin 2006) genannt. Zur Erzielung von Markenglaubwürdigkeit, Markenzufriedenheit und damit Markenvertrauen ist mindestens eine Konsistenz zwischen Markenerwartungen und Markenerlebnissen auf Nachfragerseite notwendig, die auf Anbieterseite eine Konsistenz zwischen kommuniziertem Markennutzenversprechen und tatsächlichem Markenverhalten erfordert (Piebler 2011; Burmann et al. 2012). Die Erfüllung des Markennutzenversprechens stellt damit einen zentralen Erfolgsfaktor des Markenmanagements dar (Buckley/Williams 2005).

Im Gegensatz zum Markennutzenversprechen, dessen Ausgestaltung und Kommunikation im Aufgabenbereich des Markenmanagements liegt und damit unmittelbar steuerbar ist, ist die Steuerung des Markenverhaltens schwieriger, da Nachfrager an zahlreichen Brand Touch Points das Verhalten der aktuellen Mitarbeiter als Indikator für das Markenverhalten heranziehen (Piebler 2011; Burmann et al. 2012). Damit sind aktuelle Mitarbeiter mit ihrem Verhalten im Nachfragerkontakt für die Umsetzung des Markennutzenversprechens verantwortlich (Bergstrom et al. 2002; Aaker 2004), weswegen ihnen im Markenmanagementprozess eine entscheidende Rolle zuteilwird (Harris/De Chernatony 2001; Mitchell 2002; Ind 2003). Dieser schwierigen Aufgabe widmet sich das sogenannte Internal Branding. Die Erfolgswirkung der Übereinstimmung von Markennutzenversprechen und Markenverhalten in Form des Mitarbeiterverhaltens konnte bereits empirisch nachgewiesen werden. So hat die Behavioral Branding-Qualität, d.h. das Ausmass der Markenkonformität des Mitarbeiterverhaltens, einen signifikant positiven Einfluss auf den Beitrag der Marke zum Gesamterfolg des Unternehmens (Henkel et al. 2007). Die Bedeutung des Managements des Mitarbeiterverhaltens wird in Zukunft noch steigen, da Mitarbeiter im Kontext sich angleichender und damit austauschbarerer Angebote von Unternehmen zunehmend zum eigentlichen Unterscheidungskriterium und Wettbewerbsvorteil werden (Spannagl 2005; Mosley 2007).

Sowohl das Konzept des Employer Branding als auch das Konzept des Internal Branding sind daher für den Unternehmenserfolg von entscheidender Bedeutung. Während Employer Branding für den Beschaffungsmarkt von Unternehmen in Bezug auf aktuelle und potentielle Mitarbeiter von hoher Relevanz ist, weist Internal Branding zentrale Bedeutung für die Umsetzung des Markennutzenversprechens gegenüber weiteren externen Zielgrup- 
pen (insbesondere Nachfragern) auf. Dennoch herrscht vor allem in der Unternehmenspraxis Unklarheit darüber, inwieweit Employer Branding und Internal Branding synonym zu verwenden sind oder ob die Begriffe unterschiedliche Konzepte repräsentieren. Auch in der Wissenschaft gibt es zwar zahlreiche Auseinandersetzungen mit Employer Branding und Internal Branding, allerdings nur wenige Arbeiten, die die Relation beider Konzepte betrachten. Daher ist es das Ziel des vorliegenden Artikels, die Konzepte Employer Branding und Internal Branding hinsichtlich Begriff, Zielsetzung und Zielgrössen sowie Instrumenten vorzustellen, Gemeinsamkeiten und Unterschiede zu erörtern sowie einen Ansatz zur Integration im Rahmen der identitätsbasierten Markenführung zu erarbeiten. Entgegen der zugespitzten Formulierung beim Titel des Aufsatzes handelt es sich nämlich nicht um konkurrierende, sondern um sich ergänzende Konzepte, die jedoch einer klaren Abgrenzung bedürfen, um eine sinnvolle Integration zu ermöglichen.

\section{Employer Branding vs. Internal Branding: Begriff}

Unter dem Begriff des Employer Branding wird die Anwendung von Markenmanagementprinzipien auf das HR-Management subsumiert (Backhaus/Tikoo 2004; Edwards 2010). Voraussetzung ist die Annahme, dass der Arbeitgeber als Marke interpretiert werden kann (Ambler/Barrow 1996). Es handelt sich um ein Konzept, das zunächst überwiegend in der Praxis entwickelt wurde (Backhaus/Tikoo 2004; Kucherov/Zavyalova 2012). Der Begriff des Employer Branding geht auf Ambler/Barrow (1996, 187) zurück, die die Employer Brand als „package of functional, economic and psychological benefits provided by employment, and identified with the employing company" definiert haben. Employer Branding bezieht sich demnach auf Mitarbeiter. Dabei sind in der Literatur unterschiedliche Verständnisse von Employer Branding zu finden, die sich dahingehend unterscheiden, welche Mitarbeiter im Fokus des Konzeptes stehen. So betrachten Foster et al. (2010) und Schmidt/Kilian (2012) das Konzept nur im Hinblick auf die externe Zielgruppe der potentiellen Mitarbeiter. Neben diesem engen Verständnis von Employer Branding gibt es auch ein breites Verständnis, das zusätzlich auch aktuelle Mitarbeiter als Fokus von Employer Branding umfasst (Ewing et al. 2002; Backhaus/Tikoo 2004; Moroko/Uncles 2008; Edwards 2010; Martin et al. 2011; Elving et al. 2013). Teilweise werden neben aktuellen sogar ehemalige Mitarbeiter beim Employer Branding berücksichtigt (Sponheuer 2010; Böttger 2012; Roj 2013). Aktuelle Definitionen im Rahmen des umfassenden Verständnisses betrachten Employer Branding als identitätsbasierten Markenmanagementprozess der Employer Brand, der Planung, Steuerung, Koordination, Implementierung und Kontrolle aller dazugehöriger Aktivitäten umfasst (Sponheuer 2010; Böttger 2012; Roj 2013).

Kritisch ist hierbei jedoch die Verwendung der Begriffe Employer Brand (Arbeitgebermarke), Employer Brand Identity (Arbeitgebermarkenidentität) sowie Employer Brand Image (Arbeitgebermarkenimage) zu sehen. In diesem Verständnis wird von der Arbeitgebermarke als eigenständiger Marke gesprochen. Dies impliziert, dass es sich um unterschiedliche Marken handelt, die für die Zielgruppe der Mitarbeiter und andere Zielgruppen (insbesondere Nachfrager) eingesetzt werden. Wenngleich dies für Unternehmen, die die Markenarchitekturstrategie des House of Brands verfolgen, hinsichtlich der Zielgruppen Mitarbeiter und Nachfrager zutreffen mag, gilt dies nicht für weitere potentielle Zielgruppen wie Lieferanten, Handel, Shareholder, Fremdkapitalgeber etc. Auch hinsichtlich der Zielgruppen Mitarbeiter und Nachfrager gilt die Annahme unterschiedlicher Marken nicht für Unternehmen, die beispielsweise eine Branded House-Strategie verfolgen. Den- 
noch wird im Rahmen des Employer Branding trotz der formalen Existenz von nur einer Marke zugleich von Arbeitgebermarke, Unternehmensmarke und zum Teil auch Nachfrager- beziehungsweise Konsumentenmarke gesprochen. Diese Differenzierung ist jedoch künstlich und nicht sinnvoll, da das Bezugsobjekt identisch ist. Deutlich wird dies, wenn die Markendefinition der American Marketing Association zugrunde gelegt wird, die die Marke als „name, term, design, symbol, or any other feature that identifies one seller's good or service as distinct from those of other sellers" (American Marketing Association 2013) definiert (Keller 2008; Kotler/Armstrong 2014). Der Name „Siemens“ unterscheidet sich beispielsweise nicht in Abhängigkeit davon, ob die Marke aus Mitarbeitersicht oder aus Sicht anderer Zielgruppen betrachtet wird. Auch unter Zugrundlegung der Markendefinition im identitätsbasierten Ansatz, in dem die Marke als aus Sicht relevanter Zielgruppen differenzierendes Nutzenbündel definiert wird (Burmann et al. 2003), macht eine Aufspaltung in Arbeitgebermarke, Unternehmensmarke, Konsumentenmarke etc. keinen Sinn, denn die unterschiedlichen Zielgruppen sind explizit in der Markendefinition enthalten. Es handelt sich lediglich um unterschiedliche Nutzen, die für die Zielgruppen relevant sind. Die Marke als Nutzenbündel repräsentiert alle für die relevanten Zielgruppen spezifischen Nutzen. Dementsprechend schliessen sich die Autoren hier der Ansicht von Schmidt/Kilian (2012) an, die nur wenig Sinn in der eigenständigen Betrachtung einer neben Unternehmensmarke und Produktmarken existierenden Employer Brand sehen. Employer Branding wird daher als ein Konzept verstanden, das mit der Entwicklung sowie internen und externen Umsetzung und Kontrolle des an aktuelle und potentielle Mitarbeiter gerichteten, aus der Markenidentität abgeleiteten Markennutzenversprechens befasst ist.

Des Weiteren ist im Zusammenhang mit dem Konzept des Employer Branding kritisch zu hinterfragen, inwieweit dieses ein Mehrwehrt gegenüber dem klassischen HR-Management liefert oder ob es sich nicht um ,alten Wein in neuen Schläuchen“ handelt (Ewing et al. 2002; Forster et al. 2012). Tatsächlich gibt es im klassischen HR-Management beispielsweise mit dem Personalmarketing bereits HR-Instrumente, die mit Marketingprinzipien angereichert sind. Nichtsdestotrotz ist ein Mehrwert des Employer Branding auszumachen. So ist das Employer Branding im breiten Verständnis nicht nur mit der Formulierung und Kommunikation des Markennutzenversprechens, sondern auch mit der Umsetzung befasst (Mosley 2007). Es integriert damit zahlreiche HR-Aktivitäten wie Rekrutierung, Selektion, Entwicklung und Karrieremanagement im Rahmen eines übergeordneten HR-Konzeptes (Backhaus/Tikoo 2004). Ohne diesen Umsetzungsaspekt besteht die Gefahr, dass das an Mitarbeiter abgegebene Markennutzenversprechen nicht erfüllt wird, was negative Konsequenzen wie geringe Leistung, Unzufriedenheit oder gar Abwanderung zur Folge hätte (Backhaus/Tikoo 2004; Foster et al. 2010).

Das Konzept des Internal Branding beziehungsweise die synonym verwendeten Konzepte der internen Markenführung (Brexendorf/Tomczak 2004; Piehler 2011), der innengerichteten Markenführung (Burmann/Zeplin 2004, 2005a) sowie des Behavioral Branding (Tomczak et al. 2012) tauchen bereits bei Keller (1999) sowie bei Thomson et al. (1999) auf, ohne jedoch explizit definiert zu werden. In der englischsprachigen Literatur wurde das Internal Branding-Konzept insbesondere von Burmann/Zeplin (2005b), Vallaster/De Chernatony (2005, 2006), Punjaisri/Wilson (2007, 2011), King/Grace (2008, 2012), Punjaisri et al. (2008, 2009), und Burmann et al. (2009) thematisiert beziehungsweise weiterentwickelt. Vertreter der deutschsprachigen Literatur zum Internal Branding-Konzept sind insbesondere Wittke-Kothe (2001), Brexendorf/Tomczak (2004), Brubn (2005, 2008), 
Burmann/Zeplin (2004, 2005a, 2005c), Esch et al. (2005) sowie Tomczak et al. (2012). Inhaltlich werden dabei unter Internal Branding überwiegend Massnahmen verstanden, die eine Verankerung der Marke beziehungsweise deren Identität bei den Mitarbeitern mit dem Zweck der Beeinflussung des Mitarbeiterverhaltens hin zu einem markenkonformen Verhalten zum Ziel haben. Daher wird das Konzept wie folgt definiert: Internal Branding ist ein Konzept, mit dem die Markenidentität und das auf relevante externe Zielgruppen (insbesondere Nachfrager) gerichtete Markennutzenversprechen bei den Mitarbeitern verankert wird, damit diese sich entsprechend der Markenidentität und dem Markennutzenversprechen verhalten, so dass auf Seite der externen Zielgruppen die Markenerwartungen erfüllt werden (Herrmann et al. 2002; Burmann/Zeplin 2005c; Esch et al. 2005; Piehler 2011; Batt 2013).

Sowohl der Begriff des Employer Branding als auch der Begriff des Internal Branding beziehen sich demnach auf Konzepte, die auf Mitarbeiter fokussieren. Wird das enge Verständnis von Employer Branding zugrunde gelegt, ist der Unterschied zwischen den Begriffen eindeutig: Während das Internal Branding zunächst auf aktuelle Mitarbeiter fokussiert, sind beim Employer Branding potentielle Mitarbeiter Gegenstand der Betrachtung. Wird allerdings das breite Verständnis von Employer Branding zugrunde gelegt, ist die Abgrenzung zwischen den Konzepten schwieriger, da beim breiten Employer Branding-Verständnis auch aktuelle Mitarbeiter Berücksichtigung finden. Dennoch bleibt ein zentraler Unterschied beider Konzepte erhalten. So geht es beim Internal Branding darum, ein an externe Zielgruppen (insbesondere Nachfrager) kommuniziertes Markennutzenversprechen einzulösen. Der Mitarbeiter ist in diesem Zusammenhang zwar Zielgruppe der Internal Branding-Instrumente, das finale Ziel ist jedoch auf Seite externer Zielgruppen (insbesondere bei Nachfragern) zu sehen. Demgegenüber geht es beim Employer Branding darum, ein an die Zielgruppe der Mitarbeiter zu kommunizierendes Markennutzenversprechen zu entwickeln und dieses einzulösen. Employer Branding für sich allein genommen ist damit unter Markenmanagementgesichtspunkten nicht ausreichend. Deutlich macht dies auch Mellor (1999, 26): „One problem with the employer branding approach is that it focuses on employees in isolation, without a link to customer. “ Eine Ergänzung um Internal Branding ist für den Markenerfolg notwendig.

\section{Employer Branding vs. Internal Branding: Zielsetzung und Zielgrössen}

Mitarbeiterbezogene Zielgrössen des Employer Branding sind in der Literatur auf der Ebene der finalen verhaltensbezogenen Zielgrössen, auf der Ebene verhaltensintentionsbezogener Zielgrössen sowie auf der Ebene vorgelagerter Zielgrössen zu finden. Auf diesen Ebenen kann darüber hinaus eine Differenzierung der Zielgrössen für potentielle und aktuelle Mitarbeiter vorgenommen werden.

Finale verhaltensbezogene Zielgrössen des Employer Branding sind bei potentiellen Mitarbeitern die Bewerbung (Moroko/Uncles 2008; Böttger 2012; Forster et al. 2012; Roj 2013) sowie im Falle eines Angebots seitens des Unternehmens die Zusage (Moroko/ Uncles 2008; Böttger 2012; Roj 2013). Voraussetzung dafür sind bei potentiellen Mitarbeitern die verhaltensintentionsbezogenen Zielgrössen Bewerbungsabsicht (Edwards 2010; Böttger 2012; Roj 2013) und Annahmeabsicht (Böttger 2012). Vorgelagerte zentrale Zielgrösse dieser Verhaltensintentionen stellt ein positives, differenzierendes Markenimage (als attraktiver Arbeitgeber) dar (Ewing et al. 2002; Backhaus/Tikoo 2004; Edwards 2010; Kucherov/Zavyalova 2012; Elving et al. 2013). Beim Markenimage handelt es sich um das 
„[...] in der Psyche relevanter externer Zielgruppen fest verankerte, verdichtete und wertende Vorstellungsbild von einer Marke“ (Burmann et al. 2012, 59). Eng damit verknüpft ist das Konzept der Arbeitgeberattraktivität (Ewing et al. 2002; Backhaus/Tikoo 2004; Edwards 2010; App et al. 2012; Elving et al. 2013). Grundvoraussetzung für den Aufbau eines Images ist wiederum die Bekanntheit des Arbeitgebers bzw. der Marke (Backhaus/ Tikoo 2004; Moroko/Uncles 2008; Sponhener 2010; Roj 2013).

Finale verhaltensbezogene Zielgrössen des Employer Branding sind bei aktuellen Mitarbeitern die Leistung, auch als Engagement, Einsatz oder Produktivität bezeichnet (Backhaus/Tikoo 2004; Moroko/Uncles 2008; Martin et al. 2011; Kucherov/Zavyalova 2012; Elving et al. 2013), sowie die Loyalität (Backhaus/Tikoo 2004; Moroko/Uncles 2008; Martin et al. 2011; App et al. 2012; Kucherov/Zavyalova 2012; Elving et al. 2013). Die entsprechenden verhaltensintentionsbezogenen Zielgrössen bei aktuellen Mitarbeitern sind Leistungsbereitschaft (Sponheuer 2010) und Loyalitätsabsicht bzw. Intention zu bleiben (Ewing et al. 2002; Böttger 2012). Als vorgelagerte Zielgrössen bei aktuellen Mitarbeitern werden in der Literatur der Person-Organisation-Fit bzw. der Mitarbeiter-Marke-Fit (Foster et al. 2010; Sponheuer 2010; Forster et al. 2012; Elving et al. 2013), die Zufriedenheit (Sponheuer 2010), die Motivation (Sponheuer 2010; Forster et al. 2012), die Identifikation (Backhaus/Tikoo 2004; Edwards 2010) sowie die Bindung bzw. das Commitment (Backhaus/Tikoo 2004; Barrow/Mosley 2006; Edwards 2010; Sponhener 2010; Kucherov/Zavyalova 2012; Elving et al. 2013) angeführt.

Internal Branding hat zum Ziel, ein mit Markenidentität und Markennutzenversprechen konformes Verhalten der Mitarbeiter zu erzeugen, weswegen dieses in einer Reihe wissenschaftlicher Internal Branding-Modelle die zentrale Zielgrösse darstellt. So wird vom Leben der Markenidentität und des Markenversprechens (Brexendorf/Tomczak 2004), von markenunterstützendem Mitarbeiterverhalten (Vallaster/De Chernatony 2005; Punjaisril Wilson 2011), Brand Behavior (Wentzel et al. 2012) oder Brand Citizenship Behaviour (Burmann/Zeplin 2005b; Strödter 2008; Piehler 2011; King/Grace 2012) gesprochen. Brand Citizenship Behaviour, das als aus der Organisationsforschung stammendes Konstrukt theoretisch sowie empirisch fundiert ist, wird in Anlehnung an die Definition des Organizational Citizenship Behaviour (Graham 1991; Van Dyne et al. 1994) sowie in Anlehnung an die Definition des Brand Behavior (Wentzel et al. 2012) als globales Konzept, welches alle Verhaltensweisen eines Mitarbeiters umfasst, die im Einklang mit der Markenidentität und dem Markennutzenversprechen stehen und in Summe die Marke stärken, verstanden. Als vorgelagerte mitarbeiterbezogene Zielgrössen werden in der Literatur Brand Commitment (Maloney 2007; Strödter 2008; Burmann et al. 2009; King/Grace 2010; 2012), Markenidentifikation (Punjaisri/Wilson 2007; Burmann et al. 2009; Punjaisri et al. 2009), Mitarbeiter-Marke-Fit (Maloney 2007; Strödter 2008; Morhart et al. 2009; Burmann et al. 2009; Burmann/König 2011; Piehler 2011) und Markenwissen (Piehler 2011; Esch et al. 2012a; Wentzel et al. 2012) diskutiert.

Brand Commitment stellt die psychologische Verbundenheit von Mitarbeitern mit der Marke dar (Burmann/Zeplin 2005b; Punjaisri/Wilson 2011; King/Grace 2012). Während Markenidentifikation und Mitarbeiter-Marke-Fit in einigen Modellen als Dimensionen von Brand Commitment betrachtet werden (Burmann/Zeplin 2005b; Maloney 2007; Burmann/König 2011; Piehler 2011), wird in der Organisationsforschung zwischen Commitment, Identifikation und Internalisierung unterschieden (Ashforth/Mael 1989; Gautam et al. 2004; Van Knippenberg/Sleebos 2006). Dementsprechend stellt Markenidentifikation, 
definiert als Zugehörigkeitsgefühl zur Marke, auch in einigen wissenschaftlichen Modellen des Internal Branding eine vorgelagerte Zielgrösse von markenbezogenem Mitarbeiterverhalten dar (Punjaisri et al. 2009). Mit dem Mitarbeiter-Marke-Fit liegt eine weitere vorgelagerte Internal Branding-Zielgrösse vor (Strödter 2008; Morhart et al. 2009; Esch/Strödter 2012). Schliesslich wird auch das Markenwissen der Mitarbeiter als vorgelagerte Zielgrösse in einigen wissenschaftlichen Modellen des Internal Branding angeführt. Neben der impliziten Berücksichtigung (Herrmann et al. 2002; Burmann/Zeplin 2005b; Vallaster/De Chernatony 2005; De Chernatony et al. 2006) wird Markenwissen explizit nur in wenigen wissenschaftlichen Modellen des Internal Branding erwähnt (Thomson et al. 1999; Punjaisri/Wilson 2007; Kimpakorn/Tocquer 2009; Miles et al. 2011; Piehler 2011; Wentzel et al. 2012). Dabei wird das Markenwissen der Mitarbeiter als Kenntnis und Verständnis spezifischer markenbezogener Informationen, die für ein markenkonformes Verhalten der Mitarbeiter von Relevanz sind, definiert (Piehler 2011).

Zusammenfassend ist festzuhalten, dass es auf Seite der mitarbeiterbezogenen Zielgrössen von Employer Branding und Internal Branding einige Gemeinsamkeiten gibt. So tauchen in beiden Konzepten der Fit der Mitarbeiter zur Organisation bzw. Marke sowie die Konstrukte Identifikation und Commitment auf. Wenngleich Fit, Identifikation und Commitment im Internal Branding-Konzept einen expliziten Markenfokus aufweisen, sind diese Zielgrössen des Employer Branding für das Bezugsobjekt Unternehmensmarke mit den Internal Branding-Zielgrössen Mitarbeiter-Marke-Fit, Markenidentifikation und Brand Commitment inhaltlich identisch. Der fehlende explizite Markenfokus dieser Zielgrössen in der Employer Branding-Literatur resultiert daher, dass nicht alle klassischen HR-Zielgrössen in den Markenkontext übertragen werden. Insofern liegt hier für diese Zielgrössen keine inhaltliche Divergenz zwischen den Konstrukten beider Ansätze vor, sondern lediglich eine unterschiedliche Bezeichnung, die aus der Herkunft der Forscher aus dem HRoder Markenbereich und der jeweiligen Betrachtungsperspektive resultiert.

Ein zentraler Unterschied zwischen Zielgrössen von Employer Branding und Internal Branding ist hinsichtlich der Verhaltenskonstrukte auszumachen. Während das Employer Branding auf allgemeine HR-Zielgrössen wie die Leistung fokussiert, stehen beim Internal Branding mit dem Brand Citizenship Behaviour spezifische markenbezogene Verhaltensweisen im Mittelpunkt. Brand Citizenship Behaviour ist somit wesentlich enger formuliert und umfasst nur die aus Markenidentität und Markennutzenversprechen abgeleiteten Verhaltensweisen. Des Weiteren repräsentiert das Brand Citizenship Behaviour die Verbindung des Mitarbeiterverhaltens mit externen Zielgruppen wie Nachfragern. Somit gibt es Zielgrössen, die nur beim Employer Branding Berücksichtigung finden. Hierbei handelt es sich um klassische HR-Zielgrössen, die in keiner direkten Beziehung zum Brand Citizenship Behaviour stehen und damit für Internal Branding keine direkte Bedeutung haben. Dazu gehören beispielsweise über das Brand Citizenship Behaviour hinausgehende leistungsbezogene Verhaltensweisen, Loyalität sowie die entsprechenden Verhaltensintentionen und vorgelagerte Zielgrössen wie allgemeine Arbeitszufriedenheit und Motivation. Schliesslich tauchen Zielgrössen wie Bekanntheit, Bewerbungsabsicht, Annahmeabsicht, Bewerbung und Zusage, die sich überwiegend auf potentielle Mitarbeiter beziehen, ebenfalls nur beim Employer Branding auf. Demgegenüber ist Markenwissen als Kenntnis und Verständnis spezifischer markenbezogener Informationen, die für ein markenkonformes Verhalten der Mitarbeiter von Relevanz sind, eine spezifische Internal Branding-Zielgrösse. 


\section{Employer Branding vs. Internal Branding: Instrumente}

Im Rahmen des Employer Branding kommen Instrumente des HR-Managements, der Mitarbeiterführung sowie der internen und externen Kommunikation zum Einsatz. Im Bereich des HR-Managements umfasst dies im Hinblick auf potentielle Mitarbeiter Instrumente der Personalrekrutierung wie z.B. Personalanzeigen und Recruiting-Events (Barrow/ Mosley 2006; Mosley 2007; Foster et al. 2010; Martin et al. 2011; Elving et al. 2013). Ebenfalls im Hinblick auf potentielle Mitarbeiter kommen Instrumente der Personalselektion wie z.B. Einstellungsinterviews oder Assessment Center in Frage (Foster et al. 2010). Für aktuelle Mitarbeiter können im Rahmen der Personaleinführung Einstiegstrainings oder -veranstaltungen für neue Mitarbeiter eingesetzt werden (Barrow/Mosley 2006; Mosley 2007). Weitere im Rahmen des Employer Branding genutzte Instrumente sind Massnahmen der Personalentwicklung wie z.B. Trainings, Weiterbildungs- und Führungsnachwuchsprogramme (Barrow/Mosley 2006; Mosley 2007; Foster et al. 2010; Kucherov/ Zavyalova 2012), Massnahmen der Entgelt- und Anreizgestaltung wie extrinsische (z.B. monetäre) oder intrinsische (z.B. freiheitliche Gestaltung des Arbeitsplatzes) Anreize (Barrow/Mosley 2006; Mosley 2007; Foster et al. 2010; Kucherov/Zavyalova 2012) sowie Massnahmen der Personalbeurteilung und -beförderung (Mosley 2007; Foster et al. 2010; Kucherov/Zavyalova 2012).

Als weiteres Instrument des Employer Branding wird in der Literatur die Mitarbeiterführung genannt (Barrow/Mosley 2006): „Likewise, core values and competencies can be seen as a framework for governing the everyday experience of employees through the communication and behavior of their immediate line managers and corporate leaders" (Mosley 2007, 131). Schliesslich stellen auch die an aktuelle Mitarbeiter gerichtete interne Kommunikation (Barrow/Mosley 2006; Mosley 2007; Edwards 2010) sowie die an potentielle Mitarbeiter gerichtete externe Kommunikation wie z.B. Arbeitgeber-Imagekampagnen (Edwards 2010; Foster et al. 2010) Instrumente von Employer Branding dar. Darüber hinaus nehmen Mitarbeiter auch die an weitere externe Zielgruppen (insbesondere Nachfrager) gerichteten Marketingaktivitäten wahr: „It is always worth bearing in mind that employees will probably pay as much, if not more attention to your external marketing than your target customers" (Barrow/Mosley 2006, 150).

In der Internal Branding-Literatur haben sich das markenorientierte HR-Management, die markenorientierte Führung sowie die interne und externe Markenkommunikation als Massnahmenbereiche etabliert (Burmann/Zeplin 2005b; Schmidt/Kilian 2012; Batt 2013). Ein markenorientiert ausgerichtetes HR-Management trägt entscheidend zur markenbezogenen Personalsozialisation bei (Piehler 2011). Verschiedene HR-Instrumente stehen dabei in Abhängigkeit von der Sozialisationsphase der Mitarbeiter zur Verfügung (Feldman 1976; Van Maanen 1976; Klimecki/Gmür 2005). Je nach Sozialisationsverständnis beginnt die Sozialisation bereits vor oder erst nach Eintritt in das Unternehmen (Klatetzki 2008). Wird die Vor-Eintritts-Phase als antizipatorische Sozialisation berücksichtigt, sind auch Instrumente der markenorientierten Personalrekrutierung und Personalselektion dem Internal Branding zuzurechnen (Piehler 2011). Konkrete Massnahmen umfassen markenorientierte Personalanzeigen, Einstellungsinterviews oder Assessment Center (Wittke-Kothe 2001; Burmann/Zeplin 2005b; De Chernatony et al. 2006). Die Eintritts-Phase stellt den Beginn des Sozialisationsprozesses im engeren Sinne dar. Instrument dieser Phase ist die markenorientierte Personaleinführung (Piehler 2011). Konkrete Massnahmen sind z.B. markenorientierte Einstiegstrainings und Einstiegsveranstaltungen für neue Mitarbeiter 
(Punjaisri et al. 2009; Brexendorf et al. 2012; Esch et al. 2012b). Schliesslich können in der Metamorphose-Phase die Instrumente der markenorientierten Personalentwicklung, Entgelt- und Anreizgestaltung, Personalbeurteilung und -beförderung sowie -freisetzung eingesetzt werden (Piehler 2011). Konkrete Massnahmen in diesem Bereich sind u. a. markenorientierte Trainings, Events, Coachings, Mentorenprogramme, Weiterbildungs- und Führungsnachwuchsprogramme oder markenorientierte Beförderungskriterien (Brexendorf/Tomczak 2004; Mahnert/Torres 2007; Brexendorf et al. 2012; Esch/Strödter 2012).

Die markenorientierte Führung umfasst die spezifischen Führungsstile der markenorientierten transaktionalen und transformationalen Führung sowie die markenorientierte Empowering-Führung. Bei der transaktionalen Führung werden Mitarbeitern im Rahmen der auf Austauschprozessen beruhenden leistungsorientierten Belohnung von den Führungskräften Verhaltensstandards für die Rolle als Markenrepräsentant vorgegeben und die Mitarbeiter bei Erfüllung dieser Rolle z.B. durch gute Beurteilungen, Anerkennungen, Beförderungen oder monetäre Vergütungen belohnt (Zeplin 2006; Morhart 2008). Im Gegensatz dazu versucht die transformationale Führung Werte und Einstellungen von Mitarbeitern zu beeinflussen, um ein bestimmtes Verhalten zu erzielen (Burmann/Zeplin 2005b; Morhart 2008; Morhart et al. 2012). Insbesondere das Vorleben der Markenidentität durch Führungskräfte wird in zahlreichen wissenschaftlichen Modellen des Internal Branding als zentrales Instrument gesehen (Brexendorf/Tomczak 2004; Burmann/Zeplin 2005b; Vallaster/De Chernatony 2005; De Chernatony et al. 2006; Brexendorf et al. 2012; Esch/Knörle 2012). Schliesslich ist auch die markenorientierte Empowering-Führung als auf markenorientiertes (psychologisches) Empowerment abzielende Führung durch Vorgesetzte (Piehler 2011), die über das vielfach in der Internal Branding-Literatur diskutierte strukturelle Empowerment im Sinne der Verlagerung markenbezogener Entscheidungskompetenzen auf Mitarbeiter unterer Hierarchieebenen hinausgeht (Burmann/ Zeplin 2005b; Vallaster/De Chernatony 2005; Morhart et al. 2009; Brexendorf et al. 2012; Esch/Knörle 2012), als Instrument des Internal Branding von Bedeutung. Konkrete über die Delegation von Verantwortung hinausgehende Verhaltensweisen von Führungskräften sind beispielsweise die Förderung der Entwicklung von Fähigkeiten und Fertigkeiten von Mitarbeitern, das Teilen von Informationen sowie die Interaktion mit Mitarbeitern (Arnold et al. 2000; Konczak et al. 2000).

Ein weiteres Instrument des Internal Branding stellt die interne Markenkommunikation dar, die als Teil der internen Kommunikation aufgefasst wird, der sich inhaltlich mit markenbezogenen Sachverhalten auseinandersetzt. In Anlehnung an die Definitionen der internen Kommunikation bei Brubn (1998, 2011) sollen unter interner Markenkommunikation alle Aktivitäten der Botschaftsübermittlung mit Markenbezug zwischen Mitarbeitern einer Organisation auf unterschiedlichen hierarchischen Ebenen verstanden werden. Der internen Markenkommunikation wird in zahlreichen Studien zum Internal Branding eine zentrale Bedeutung beim Aufbau von Markenwissen, Markenidentifikation und Brand Commitment beigemessen (Burmann/Zeplin 2005b; De Chernatony et al. 2006; Punjaisri et al. 2009; Hartmann 2010; Brexendorf et al. 2012; Esch et al. 2012c). Als Instrumente der internen Markenkommunikation stehen alle Instrumente der internen Kommunikation wie z.B. Intranet, Mitarbeiterzeitschrift, Workshop, E-Mail oder Mitarbeitergespräch zur Verfügung (Brubn 2011). Unter externer Markenkommunikation werden alle Aktivitäten der Botschaftsübermittlung mit Markenbezug zwischen einer markenführenden Organisation und ihren externen Zielgruppen verstanden. Im Gegensatz zur internen Markenkom- 
munikation, die nur in Ausnahmefällen direkt von externen Zielgruppen wahrgenommen wird, nehmen Mitarbeiter sehr viel häufiger externe Markenkommunikation wahr. Dementsprechend findet die externe Markenkommunikation auch im Kontext des Internal Branding als Instrument Berücksichtigung (Burmann/Zeplin 2005b; De Chernatony et al. 2006; Henkel 2008, 2009; Brexendorf et al. 2012; Henkel et al. 2012). Als Instrumente der externen Markenkommunikation stehen alle gängigen Instrumente der externen Kommunikation wie z.B. klassische Werbung, Out-of-Home-Medien, Online-Kommunikation inklusive sozialer Medien, Direktkommunikation, Public Relations, Verkaufsförderung, Messen und Ausstellungen, Event Marketing, Sponsoring sowie Product Placement zur Verfügung (Meffert et al. 2012).

Auf operativer Umsetzungsebene sind die grössten Gemeinsamkeiten zwischen Employer Branding und Internal Branding auszumachen. So werden in der Literatur für beide Konzepte Instrumente aus den Bereichen HR-Management, Führung sowie interne und externe Kommunikation angeführt. Dies ist insofern wenig überraschend, da sowohl Employer Branding als auch in einem ersten Schritt Internal Branding auf die Zielgruppe der Mitarbeiter fokussieren.

\section{Integration von Employer Branding und Internal Branding im Rahmen der identitätsbasierten Markenführung}

Zusammenfassend sind der Begriff, die Zielgrössen sowie die Instrumente von Employer Branding und Internal Branding in Abbildung 1 dargestellt.

Da, wie in Kapitel 2 dargestellt, die Betrachtung einer Employer Brand als eigenständige Marke nicht sinnvoll ist, ist auch kein eigenständiger Markenmanagementprozess zu durchlaufen. Vielmehr sind die Prozessschritte, die in der Employer Branding- und Internal Branding-Literatur genannt werden, in den allgemeinen Markenmanagementprozess einzuordnen. Hierfür eignet sich insbesondere der Managementprozess der identitätsbasierten Markenführung (Burmann et al. 2012). Dieser basiert auf einem ganzheitlichen Markenund Markenmanagementverständnis, das nicht nur Nachfrager, sondern auch weitere Stakeholder wie Mitarbeiter als zentrale Zielgruppe berücksichtigt. Damit eignet sich die auf Kapferer (1992), Keller (1993), Aaker (1996) und Meffert/Burmann (1996) zurückgehende identitätsbasierte Markenführung insbesondere auch als Grundlage für die Integration von Employer Branding und Internal Branding. So geht die identitätsbasierte Markenführung über die einseitige Ausrichtung auf die Wahrnehmung der Marke bei Nachfragern hinaus und erweitert die primär absatzmarktbezogene Sichtweise um eine Betrachtung aller für die Marke relevanten Zielgruppen (vgl. Abbildung 2).

Dem Markenimage als Fremdbild der Marke aus Sicht externer Zielgruppen steht die Markenidentität als Selbstbild der Marke aus Sicht interner Zielgruppen gegenüber. Die Markenidentität „[...] als diejenigen raum-zeitlich gleichartigen Merkmale der Marke, die aus Sicht der internen Zielgruppen in nachhaltiger Weise den Charakter der Marke prägen“ (Burmann et al. 2003, 5), stellt den Kern der identitätsbasierten Markenführung dar. Sie bringt die wesensprägenden Merkmale der Marke zum Ausdruck und stellt ein Führungskonzept dar (Burmann et al. 2012; Esch 2012). Employer Branding und Internal Branding können in den Managementprozess der identitätsbasierten Markenführung, der die Aufgaben der Markenführung strukturiert, integriert werden. Grundsätzlich werden hierbei das strategische Markenmanagement, das operative Markenmanagement sowie das Marken-Controlling unterschieden (vgl. Abbildung 3). 


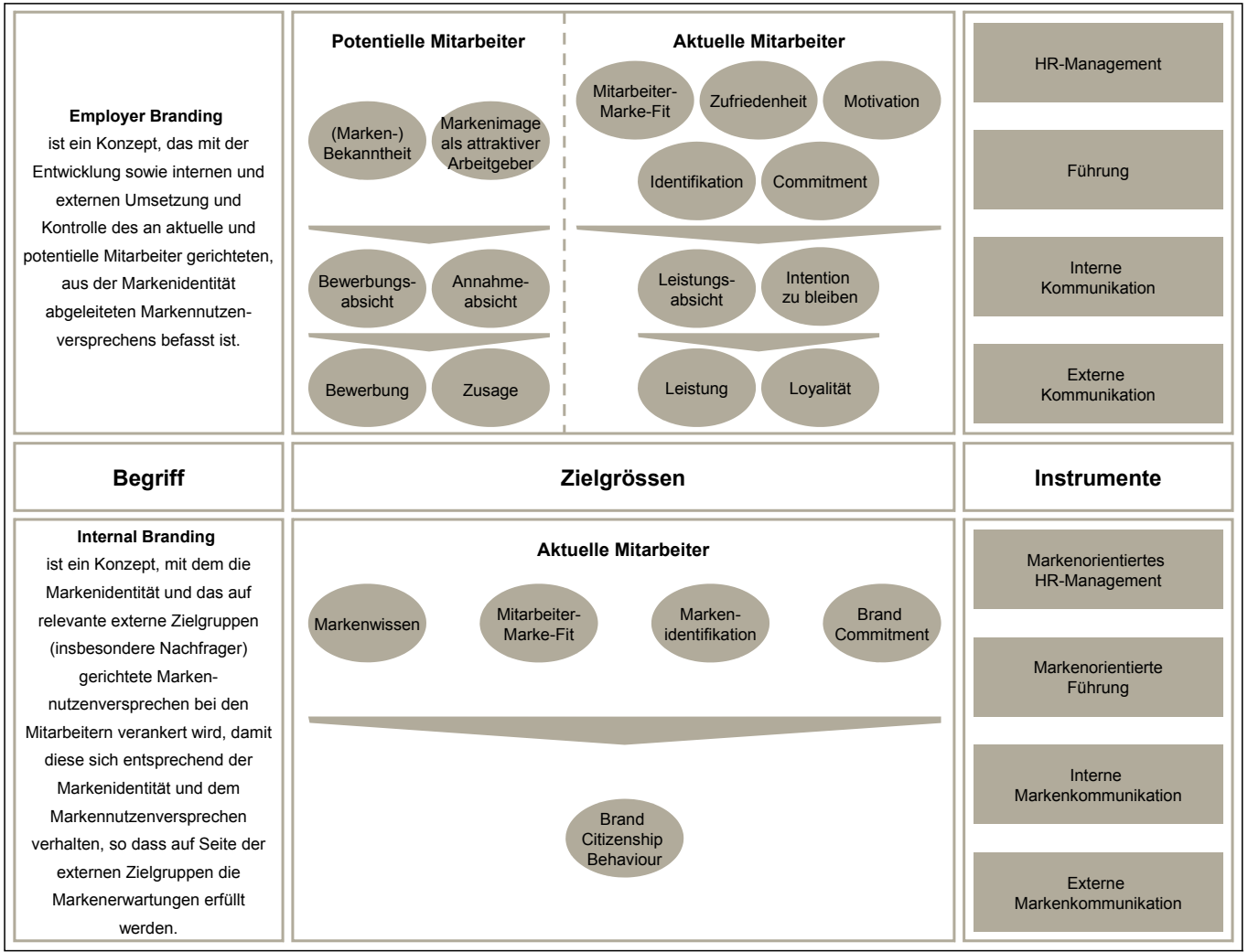

Abbildung 1: Employer Branding vs. Internal Branding

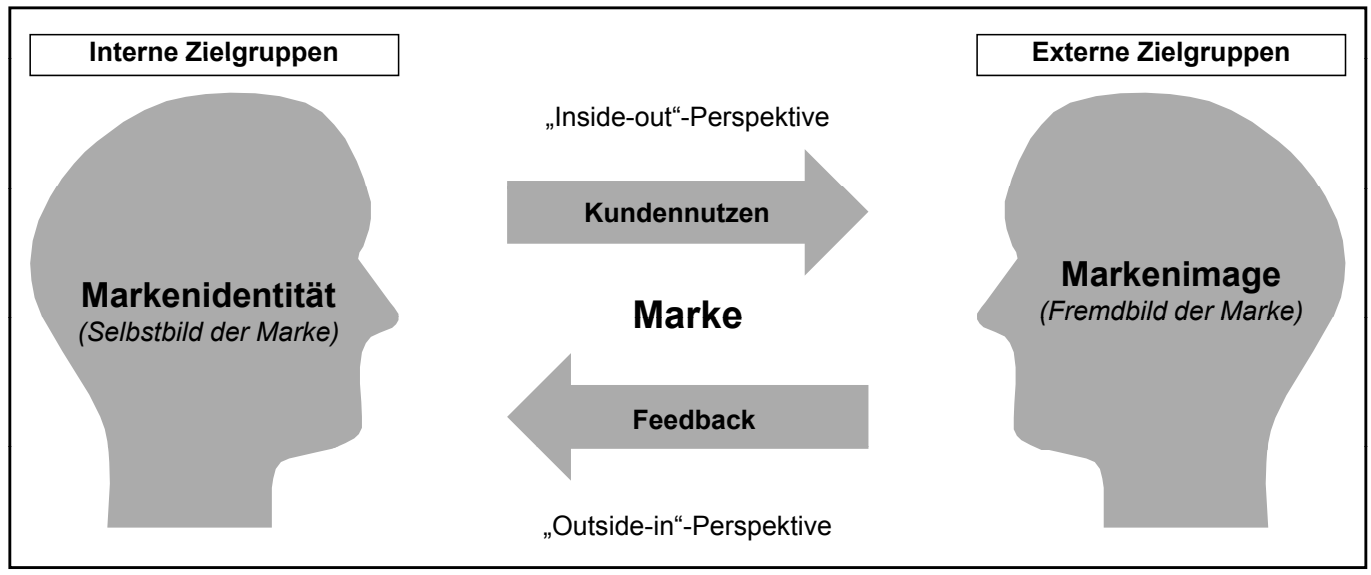

Abbildung 2: Grundkonzept der identitätsbasierten Markenführung

Quelle: Burmann et al. $(2012,29)$ 


\section{Strategisches Markenmanagement}

\section{Operatives Markenmanagement}

\section{Marken-Controlling}

Abbildung 3: Managementprozess der identitätsbasierten Markenführung

Quelle: In Anlehnung an Burmann et al. (2012, 92)

Zunächst ist im Rahmen des strategischen Markenmanagements eine Situationsanalyse vorzunehmen, die eine fundierte Analyse der Ausgangssituation darstellt und die unternehmensexterne und unternehmensinterne Faktoren berücksichtigt (Burmann et al. 2012; Meffert et al. 2012). Dazu zählen entsprechend auch Informationen, die für Employer Branding und Internal Branding von Relevanz sind, z.B. das aktuelle Image der Marke bei potentiellen und aktuellen Mitarbeitern, das Markenimage von Wettbewerbern, die Relevanz spezifischer Nutzen für die Zielgruppe der Mitarbeiter sowie das Markenwissen, der Mitarbeiter-Marke-Fit, die Markenidentifikation, das Brand Commitment und das Brand Citizenship Behaviour der Mitarbeiter. Im Anschluss daran sind die Markenziele festzulegen, die ökonomische und psychographische Zielgrössen umfassen können (Burmann et al. 2012). Auch hier sind im Hinblick auf die Zielgruppe der Mitarbeiter die bereits im Rahmen der Situationsanalyse angesprochenen mitarbeiterbezogenen Ziele sowie organisationsbezogene Ziele wie Effizienzziele für die Personalbeschaffung oder Fluktuationsraten festzulegen.

Der zentrale Prozessschritt des strategischen Markenmanagements ist die Entwicklung der Markenidentität. Da die Markenidentität das Selbstbild der Marke repräsentiert, sind keine zielgruppenspezifischen Identitäten zu entwickeln. Zielgruppen finden erst wieder bei der Markenpositionierung Berücksichtigung, bei der die Komponenten der Markenidentität in zielgruppenspezifische funktionale und symbolische Markennutzenversprechen verdichtet und übersetzt werden (Burmann et al. 2012). Dabei sollten die Markennutzenversprechen für die jeweilige Zielgruppe verständlich, (kaufverhaltens-)relevant und gegenüber konkurrierenden Marken überlegen sein (Burmann et al. 2012). Beinhaltet eine Markenidentität beispielsweise den Wert innovativ, kann eine Übersetzung in ein Markennutzenversprechen gegenüber Nachfragern z.B. im Angebot innovativer Produkte mit neuen Technologien erfolgen. Die entsprechende Übersetzung in ein Markennutzenversprechen gegenüber Mitarbeitern stellt z.B. das Angebot moderner, flexibler Arbeitszeitregelungen dar. Während Gehalt, faires Bonussystem, sichere Arbeitsplätze, Arbeitsinhalt, die Möglichkeit, international tätig zu sein, Karriereperspektiven und Entwicklungsperspektiven potentielle funktionale Markennutzen für die Zielgruppe der Mitarbeiter darstellen, sind 
Prestige und Reputation, eine starke Kultur, positive interpersonelle Beziehungen und Teamarbeit potentielle symbolische Markennutzen für die Zielgruppe der Mitarbeiter (Backhaus/Tikoo 2004; Lievens 2007; Mosley 2007; Burmann et al. 2012; Kucherov/ Zavyalova 2012). Markennutzenversprechen sollten für alle relevanten Stakeholder, die dann als Zielgruppe im Rahmen der Marktbearbeitung berücksichtigt werden, erarbeitet werden. Dies bedeutet unter Umständen, dass über Markennutzenversprechen für Nachfrager und Mitarbeiter hinaus auch adaptierte Markennutzenversprechen für Handel, Investoren, Umweltorganisationen etc. zu erarbeiten sind. Da die Basis der Markennutzenversprechen die Markenidentität darstellt, werden diese unterschiedlichen Formen des Markennutzenversprechens gegenüber verschiedenen Zielgruppen sich in weiten Teilen überlappen. Schliesslich ist im Kontext der Markenbudgetierung über die Verteilung des Budgets zu entscheiden (Burmann et al. 2012). Hier ist nicht nur festzulegen, wie das Budget auf verschiedene Marken zu verteilen ist, sondern im Hinblick auf Employer Branding und Internal Branding auch, wie das Budget auf die Zielgruppen zu verteilen ist.

Im Anschluss an das strategische Markenmanagement sind die strategischen Entscheidungen im operativen Markenmanagement umzusetzen (vgl. Abbildung 4). Im Rahmen des Employer Branding erfolgt die Umsetzung des Markennutzenversprechens gegenüber aktuellen und potentiellen Mitarbeitern. Hier stehen die bereits vorgestellten Instrumente des HR-Managements, der Führung und der internen sowie externen Kommunikation zur Verfügung. Durch das External Branding erfolgt die Umsetzung des Markennutzenversprechens gegenüber weiteren externen Zielgruppen (insbesondere Nachfragern). Hier ste-

\section{Operatives Markenmanagement}

\begin{tabular}{c|c}
\hline Internal Branding & Employer Branding \\
Sicherstellung der & Umsetzung des für \\
Umsetzung des für & aktuelle und potentielle \\
\hline Nachfrager, Lieferanten, & Mitarbeiter spezifizierten \\
Handel, Kapitalgeber etc. & Markennutzenversprechens \\
spezifizierten & \\
Markennutzenversprechens \\
durch aktuelle Mitarbeiter
\end{tabular}

\section{External Branding}

Umsetzung des für Nachfrager, Lieferanten, Handel, Kapitalgeber etc. spezifizierten Markennutzenversprechens

Marktgerichtete Instrumente (Marketing-Mix: Produkt, Preis, Distribution, Organisationsgerichtete Instrumente externe Kommunikation)

(HR-Management, Führung, interne Kommunikation) 
hen die Instrumente des Marketing-Mix zur Verfügung. Im Rahmen des Internal Branding ist die Umsetzung des Markennutzenversprechens gegenüber externen Zielgruppen (insbesondere Nachfragern) durch die Mitarbeiter sicherzustellen (Brand Citizenship Behaviour). Als Instrumente kommen insbesondere das markenorientierte HR-Management, die markenorientierte Führung sowie die interne und externe Markenkommunikation in Betracht. Grundsätzlich ist auf Ebene der Markenmanagementinstrumente zur Umsetzung von Internal Branding, Employer Branding und External Branding zwischen organisationsgerichteten und marktgerichteten Instrumenten zu unterscheiden. Während Internal Branding sich überwiegend organisationsgerichteter Instrumente bedient, nutzt Employer Branding organisationsgerichtete Instrumente für aktuelle Mitarbeiter sowie marktgerichtete Instrumente für potentielle Mitarbeiter. Beim External Branding werden wiederum überwiegend marktgerichtete Instrumente eingesetzt.

Das Markencontrolling umfasst schliesslich die Informationsversorgung und Beratung aller mit der Markenführung befassten Stellen und übernimmt eine übergeordnete Koordinationsfunktion zur Unterstützung der markenspezifischen Planungs-, Steuerungs- und Kontrollprozesse im Unternehmen (Burmann et al. 2012). Im Hinblick auf Employer Branding und Internal Branding sind auch die Erreichung der im strategischen Markenmanagement festgelegten mitarbeiterbezogenen Ziele sowie deren operative Umsetzung zu prüfen.

Wenngleich insbesondere hinsichtlich der Verantwortlichkeiten für Internal Branding in Unternehmen noch Forschungsbedarf besteht, kann grundsätzlich der Personalabteilung die Hauptverantwortlichkeit für Employer Branding und der Marketing- bzw. Markenmanagementabteilung die Hauptverantwortlichkeit für External Branding zugesprochen werden. Für das Internal Branding stehen beide Bereiche gemeinsam in Verantwortung. Existiert für das External Branding neben einer Marketingabteilung eine eigenständige Markenmanagementabteilung, ist letztere insbesondere mit dem strategischen Markenmanagement, d.h. der Festlegung der Markenziele, der Markenidentität, der Markenpositionierung etc., sowie dem Marken-Controlling befasst. Die Marketingabteilung würde dann insbesondere die Aufgaben des operativen Markenmanagements, d.h. die Umsetzung der strategischen Entscheidungen, übernehmen.

Hinsichtlich der konkreten organisationalen Umsetzung in Unternehmen liegen strategische Employer Branding-Entscheidungen im Aufgabenbereich der Personalabteilung, die dabei von der Markenmanagementabteilung unterstützt wird. Dies umfasst beispielsweise die Festlegung der über die Internal Branding-Ziele hinausgehenden Employer BrandingZiele sowie die Entwicklung des aus der Markenidentität abgeleiteten Markennutzenversprechens für aktuelle und potentielle Mitarbeiter. Demgegenüber ist die Markenmanagementabteilung für die Entwicklung der Markenidentität zuständig. Durch diese klare Trennung der Verantwortlichkeiten soll sichergestellt werden, dass es nicht zu einer Entwicklung einer eigenständigen „Employer Brand“ durch die Personalabteilung kommt. Die Verantwortung für das operative Employer Branding, d.h. die Umsetzung in Massnahmen des HR-Managements, der Führung sowie der internen und externen Kommunikation liegt wiederum bei der Personalabteilung. Schliesslich ist von dieser auch der Erfolg im Rahmen des Controllings der Employer-Branding-Ziele und -Massnahmen zu überprüfen.

In Bezug auf das Internal Branding ist eine klare organisatorische Abgrenzung der Aufgaben am schwierigsten. Die Markenmanagementabteilung ist insbesondere mit der Festlegung der über das Employer Branding hinausgehenden Ziele wie Markenwissen und 
Brand Citizenship Behaviour befasst. Demgegenüber werden gemeinsame Ziele von Employer Branding und Internal Branding wie Mitarbeiter-Marke-Fit, Markenidentifikation und Brand Commitment von beiden Abteilungen gemeinsam festgelegt. Auf operativer Ebene werden die organisationsgerichteten Instrumente HR-Management und Führung von der Personalabteilung mit Unterstützung der Marketingabteilung und die interne Kommunikation von der Marketingabteilung mit Unterstützung der Personalabteilung verantwortet. Marktgerichtete Instrumente liegen hingegen im Aufgabenbereich der Marketingabteilung. Hinsichtlich des Controllings ist jeweils die Abteilung zuständig, die für die jeweiligen Zielgrössen und Massnahmen primär verantwortlich ist.

Deutlich wird bei der Integration des Employer Branding und des Internal Branding in den Managementprozess der identitätsbasierten Markenführung, dass sich durch die Stakeholder- und Zielgruppenbetrachtung die Komplexität des Managements erhöht. Dies erfordert eine enge Zusammenarbeit insbesondere zwischen der Personal- und Marketingbzw. Markenmanagementfunktion. Für den Erfolg von Employer Branding und Internal Branding muss eine institutionalisierte Koordination existieren, damit beide Konzepte optimal miteinander vernetzt werden. Gelingt diese Koordination, kann die Effizienz der Markenführung erhöht und die Unternehmensmarke gestärkt werden: „Aligning internal branding with employer branding should in theory lead to a closer working relationship between the HR and marketing functions and ultimately an increase in organisational performance and a strengthened corporate brand" (Foster et al. 2010, 404).

\section{Literaturhinweise}

Aaker, D.A. (1996): Building strong brands, New York, NY et al.

Aaker, D.A. (2004): Leveraging the corporate brand, in: California Management Review, Jg. 46, Nr. 3, S. 6-18.

Ambler, T./Barrow, S. (1996): The employer brand, in: Journal of Brand Management, Jg. 4, Nr. 3, S. 185-206.

American Marketing Association (2013): Stichwort „Brand“, unter http://www.marketingpower.co $\mathrm{m} /$ layouts/Dictionary.aspx?dLetter=B (5.5.2013).

App, S./Merk, J./Büttgen, M. (2012): Employer Branding: Sustainable HRM as a competitive advantage in the market for high-quality employees, in: Management Revue, Jg. 23, Nr. 3, S. 262-278.

Arnold, J.A./Arad, S./Rhoades, J.A./Drasgow, F. (2000): The Empowering Leadership Questionnaire: The construction and validation of a new scale for measuring leader behaviors, in: Journal of Organizational Behavior, Jg. 21, Nr. 3, S. 249-269.

Arthur Jr., W./Bell, S.T./Villado, A.J./Doverspike, D. (2006): The use of person-organization fit in employment decision making: An assessment of its criterion-related validity, in: Journal of Applied Psychology, Jg. 91, Nr. 4, S. 786-801.

Ashforth, B.E./Mael, F. (1989): Social identity theory and the organization, in: Academy of Management Review, Jg. 14, Nr. 1, S. 20-39.

Backhaus, K./Tikoo, S. (2004): Conceptualizing and researching employer branding, in: Career Development International, Jg. 9, Nr. 5, S. 501-517.

Barney, J.B. (1991): Firm resources and sustained competitive advantage, in: Journal of Management, Jg. 17, Nr. 1, S. 99-120.

Barrow, S./Mosley, R. (2006): The employer brand: Bringing the best of brand management to people at work, Chichester. 
Batt, V. (2013): Qualität der Internen Markenführung, Wiesbaden.

Bergstrom, A./Blumenthal, D./Crothers, S. (2002): Why internal branding matters: The case of Saab, in: Corporate Reputation Review, Jg. 5, Nr. 2/3, S. 133-142.

Berthon, P./Ewing, M./Hah, L.L. (2005): Captivating company: Dimensions of attractiveness in employer branding, in: International Journal of Advertising, Jg. 24, Nr. 2, S. 151-172.

Böttger, E. (2012): Employer Branding, Wiesbaden.

Brexendorf, T.O./Tomczak, T. (2004): Interne Markenführung, in: Albers, S./Hassmann, V./Tomcz$a k, T$. (Hrsg.): Verkauf: Kundenmanagement, Vertriebssteuerung, E-Commerce, Digitale Fachbibliothek, Düsseldorf, S. 1-26.

Brexendorf, T./Tomczak, T./Kernstock, J./Henkel, S./Wentzel, D. (2012): Der Einsatz von Instrumenten zur Förderung von Brand Behavior, in: Tomczak, T./Esch, F.-R./Kernstock, J./Herrmann, A. (Hrsg.): Behavioral Branding: Wie Mitarbeiterverhalten die Marke stärkt, 3. Aufl., Wiesbaden, S. 337-371.

Brubn, M. (1998): Interne Kommunikation, in: Meyer, A. (Hrsg.): Grundlagen und Rahmenbedingungen des Dienstleistungs-Marketing, Managementaspekte von Dienstleistungsanbietern, programmatische Aspekte des externen Marketing, programmatische Aspekte des internen Marketing, Stuttgart, S. 1045-1062.

Bruhn, M. (2005): Interne Markenbarometer - Konzept und Gestaltung, in: Esch, F.-R. (Hrsg.): Moderne Markenführung, 4. Aufl., Wiesbaden, S. 1037-1060.

Brubn, M. (2008): Der Einfluss der Mitarbeitenden auf den Markenerfolg - Konzeptualisierung und Operationalisierung Interner Markenbarometer, in: Bauer, H.H./Huber, F./Albrecht, C. (Hrsg.): Erfolgsfaktoren der Markenführung: Know-how aus Forschung und Management, München, S. 159-177.

Bruhn, M. (2011): Unternehmens- und Marketingkommunikation, 2. Aufl., München.

Buckley, E./Williams, M. (2005): Internal branding, in: Tybout, A.M./Calkins, T. (Hrsg.): Kellogg on branding, Hoboken, NJ, S. 320-326.

Burmann, C./Blinda, L./Nitschke, A. (2003): Konzeptionelle Grundlagen des identitätsbasierten Markenmanagements, Arbeitspapier Nr. 1, Lehrstuhl für innovatives Markenmanagement, Universität Bremen, Bremen.

Burmann, C./Halaszovich, T./Hemmann, F. (2012): Identitätsbasierte Markenführung, Wiesbaden.

Burmann, C./König, V. (2011): Does internal brand management really drive brand commitment in shared-service call centers?, in: Journal of Brand Management, Jg. 18, Nr. 6, S. 374-393.

Burmann, C./Meffert, H. (2005): Managementkonzept der identitätsorientierten Markenführung, in: Meffert, H./Burmann, C./Koers, M. (Hrsg.): Markenmanagement - Grundfragen der identitätsorientierten Markenführung, 2. Aufl., Wiesbaden, S. 73-114.

Burmann, C./Meffert, H./Koers, M. (2005): Stellenwert und Gegenstand des Markenmanagements, in: Meffert, H./Burmann, C./Koers, M. (Hrsg.): Markenmanagement - Grundfragen der identitätsorientierten Markenführung, 2. Aufl., Wiesbaden, S. 3-17.

Burmann, C./Zeplin, S. (2004): Innengerichtetes identitätsbasiertes Markenmanagement - State-ofthe-Art und Forschungsbedarf, Arbeitspapier Nr. 7, Lehrstuhl für innovatives Markenmanagement, Universität Bremen, Bremen.

Burmann, C./Zeplin, S. (2005a): Innengerichtetes identitätsbasiertes Markenmanagement, in: Meffert, H./Burmann, C./Koers, M. (Hrsg.): Markenmanagement - Grundfragen der identitätsorientierten Markenführung, 2. Aufl., Wiesbaden, S. 113-138. 
Burmann, C./Zeplin, S. (2005b): Building brand commitment: A behavioural approach to internal brand management, in: Journal of Brand Management, Jg. 12, Nr. 4, S. 279-300.

Burmann, C./Zeplin, S. (2005c): Innengerichtete Markenkommunikation, in: Esch, F.-R. (Hrsg.): Moderne Markenführung, 4. Aufl., Wiesbaden, S. 1021-1036.

Burmann, C./Zeplin, S./Riley, N.-M. (2009): Key determinants of internal brand management success: An exploratory empirical analysis, in: Journal of Brand Management, Jg. 16, Nr. 4, S. 264-284.

Carless, S.A. (2005): Person-job fit versus person-organization fit as predictors of organizational attraction and job acceptance intentions: A longitudinal study, in: Journal of Occupational and Organizational Psychology, Jg. 78, Nr. 3, S. 411-429.

Chapman, D.S./Uggerslev, K.L./Carroll, S.A./Piasentin, K.A./Jones, D.A. (2005): Applicant attraction to organizations and job choice: A meta-analytic review of the correlates of recruiting outcomes, in: Journal of Applied Psychology, Jg. 90, Nr. 5, S. 928-944.

De Chernatony, L./Cottam, S./Segal-Horn, S. (2006): Communicating services brands' values internally and externally, in: Service Industries Journal, Jg. 26, Nr. 8, S. 819-836.

De Chernatony, L./Drury, S./Segal-Horn, S. (2003): Building a services brand: Stages, people and orientations, in: Service Industries Journal, Jg. 23, Nr. 3, S. 1-21.

Delgado-Ballester, E./Munuera-Alemán, J.L./Yagüe-Guillén, M.J. (2003): Development and validation of a brand trust scale, in: International Journal of Market Research, Jg. 45, Nr. 1, S. 35-53.

Dutton, J.E./Dukerich, J.M./Harquail, C.V. (1994): Organizational images and member identification, in: Administrative Science Quarterly, Jg. 39, Nr. 2, S. 239-263.

Edwards, M.R. (2010): An integrative review of employer branding and OB theory, in: Personnel Review, Jg. 39, Nr. 1, S. 5-23.

Eisend, M. (2003): Glaubwürdigkeit in der Marketingkommunikation: Konzeption, Einflussfaktoren und Wirkungspotenzial, Wiesbaden.

Elving, W.J.L./Westhoff, J.J.C./Meeusen, K./Schoonderbeek, J. (2013): The war for talent? The relevance of employer branding in job advertisements for becoming an employer of choice, in: Journal of Brand Management, Jg. 20, Nr. 5, S. 355-373.

Esch, F.-R. (2012b): Strategie und Technik der Markenführung, 7. Aufl., München.

Esch, F.-R./Fischer, A./Strödter, K. (2012a): Abstrakte Markenwerte in konkretes Verhalten übersetzen, in: Tomczak, T./Esch, F.-R./Kernstock, J./Herrmann, A. (Hrsg.): Behavioral Branding: Wie Mitarbeiterverhalten die Marke stärkt, 3. Aufl., Wiesbaden, S. 161-180.

Esch, F.-R./Fischer, A./Strödter, K. (2012c): Interne Kommunikation zum Aufbau von Markenwissen bei den Mitarbeitern, in: Tomczak, T./Esch, F.-R./Kernstock, J./Herrmann, A. (Hrsg.): Behavioral Branding: Wie Mitarbeiterverhalten die Marke stärkt, 3. Aufl., Wiesbaden, S. 101-120.

Esch, F.-R./Hartmann, K./Strödter, K. (2012a): Analyse und Stärkung des Markencommitment in Unternehmen, in: Tomczak, T./Esch, F.-R./Kernstock, J./Herrmann, A. (Hrsg.): Behavioral Branding: Wie Mitarbeiterverhalten die Marke stärkt, 3. Aufl., Wiesbaden, S. 121-139.

Esch, F.-R./Knörle, C. (2012): Führungskräfte als Markenbotschafter, in: Tomczak, T./Esch, F.-R./ Kernstock, J./Herrmann, A. (Hrsg.): Behavioral Branding: Wie Mitarbeiterverhalten die Marke stärkt, 3. Aufl., Wiesbaden, S. 373-387.

Esch, F.-R./Rutenberg, J./Strödter, K./Vallaster, C. (2005): Verankerung der Markenidentität durch Behavioral Branding, in: Esch, F.-R. (Hrsg.): Moderne Markenführung, 4. Aufl., Wiesbaden, S. 985-1008. 
Esch, F.-R./Strödter, K. (2012): Aufbau des Markencommitment in Abhängigkeit des MitarbeiterMarken-Fits, in: Tomczak, T./Esch, F.-R./Kernstock, J./Herrmann, A. (Hrsg.): Behavioral Branding: Wie Mitarbeiterverhalten die Marke stärkt, 3. Aufl., Wiesbaden, S. 141-159.

Ewing, M.T./Pitt, L.F./de Bussy, N.M./Berthon, P. (2002): Employment branding in the knowledge economy, in: International Journal of Advertising, Jg. 21, Nr. 1, S. 3-22.

Feldman, D.C. (1976): A contingency theory of socialization, in: Administrative Science Quarterly, Jg. 21, Nr. 3, S. 433-452.

Forster, A./Erz, A./Jenewein, W. (2012): Employer Branding, in: Tomczak, T./Esch, F.-R./Kernstock, J./Herrmann, A. (Hrsg.): Behavioral Branding: Wie Mitarbeiterverhalten die Marke stärkt, 3. Aufl., Wiesbaden, S. 277-294.

Foster, C./Punjaisri, K./Cheng, R. (2010): Exploring the relationship between corporate, internal and employer branding, in: Journal of Product \& Brand Management, Jg. 19, Nr. 6, S. 401-409.

Fulmer, I.S./Gerhart, B./Scott, K.S. (2003): Are the 100 best better? An empirical investigation of the relationship between being a "great place to work" and firm performance, in: Personnel Psychology, Jg. 56, Nr. 4, S. 965-993.

Gautam, T./Van Dick, R./Wagner, U. (2004): Organizational identification and organizational commitment: Distinct aspects of two related concepts, in: Asian Journal of Social Psychology, Jg. 7, Nr. 3, S. 301-315.

Graham, J.W. (1991): An essay on organizational citizenship behavior, in: Employee Responsibilities and Rights Journal, Jg. 4, Nr. 4, S. 249-270.

Harris, F./De Chernatony, L. (2001): Corporate branding and corporate brand performance, in: European Journal of Marketing, Jg. 35, Nr. 3/4, S. 441-456.

Hartmann, K. (2010): Wirkung der Markenwahrnehmung auf das Markencommitment von Mitarbeitern: Eine empirische Untersuchung der Wirkung von Markenimage, interner Kommunikation und Fit zwischen persönlichen und Markenwerten auf das Commitment, Hamburg.

Henkel, S. (2008): Werbung als Verhaltensvorbild für Mitarbeiter: Eine empirische Untersuchung am Beispiel UBS, Hamburg.

Henkel, S./Tomczak, T./Heitmann, M./Herrmann, A. (2007): Managing brand consistent employee behaviour: Relevance and managerial control of behavioural branding, in: Journal of Product $\&$ Brand Management, Jg. 16, Nr. 5, S. 310-320.

Henkel, S./Tomczak, T./Jenewein, W. (2012): Werbung als Verhaltensvorbild für Mitarbeiter, in Tomczak, T./Esch, F.-R./Kernstock, J./Herrmann, A. (Hrsg.): Behavioral Branding: Wie Mitarbeiterverhalten die Marke stärkt, 3. Aufl., Wiesbaden, S. 443-467.

Henkel, S./Wentzel, D./Tomczak, T. (2009): Die Rolle der Werbung in der internen Markenführung, in: Marketing ZFP, Jg. 31, Nr. 1, S. 43-56.

Herrmann, A./Huber, F./Wittke-Kothe, C. (2002): Interne Markenführung - Verankerung der Markenidentität im Mitarbeiterverhalten, in: Journal für Betriebswirtschaft, Jg. 52, Nr. 5/6, S. 186-205.

Homburg, C./Becker, A./Hentschel, F. (2010): Der Zusammenhang zwischen Kundenzufriedenheit und Kundenbindung, in: Brubn, M./Homburg, C. (Hrsg.): Handbuch Kundenbindungsmanagement, 7. Aufl., Wiesbaden, S. 103-134.

Homburg, C./Brubn, M. (2010): Kundenbindungsmanagement - Eine Einführung in die theoretischen und praktischen Problemstellungen, in: Brubn, M./Homburg, C. (Hrsg.): Handbuch Kundenbindungsmanagement, 7. Aufl., Wiesbaden, S. 3-39. 
Homburg, C./Koschate, N./Becker, A. (2005): Messung von Markenzufriedenheit und Markenloyalität, in: Esch, F.-R. (Hrsg.): Moderne Markenführung, 4. Aufl., Wiesbaden, S. 1393-1408.

Huber, F./Regier, S./Vollhardt, K. (2006): Determinanten des Markenvertrauens und dessen Einfluss auf die Markenloyalität - Eine kausalanalytische Studie, in: Bauer, H.H./Neumann, M.M./Schüle, A. (Hrsg.): Konsumentenvertrauen: Konzepte und Anwendungen für ein nachhaltiges Kundenbindungsmanagement, München, S. 235-247.

Ind, N. (2003): Inside out: How employees build value, in: Journal of Brand Management, Jg. 10, Nr. 6, S. 393-402.

Judge, T.A./Cable, D.M. (1997): Applicant personality, organizational culture, and organizational attraction, in: Personnel Psychology, Jg. 50, Nr. 2, S. 359-394.

Kapferer, J.-N. (1992): Die Marke - Kapital des Unternehmens, Landsberg/Lech.

Keller, K.L. (1993): Conceptualizing, measuring, and managing customer-based brand equity, in: Journal of Marketing, Jg. 57, Nr. 1, S. 1-22.

Keller, K.L. (1999): Brand mantras: Rationale, criteria and examples, in: Journal of Marketing Management, Jg. 15, Nr. 1-3, S. 43-51.

Keller, K.L. (2008): Strategic brand management, 3. Aufl., Upper Saddle River, NJ.

Kimpakorn, N./Tocquer, G. (2009): Employees' commitment to brands in the service sector: Luxury hotel chains in Thailand, in: Journal of Brand Management, Jg. 16, Nr. 8, S. 532-544.

King, C./Grace, D. (2008): Internal branding: Exploring the employee's perspective, in: Journal of Brand Management, Jg. 15, Nr. 5, S. 358-372.

King, C./Grace, D. (2010): Building and measuring employee-based brand equity, in: European Journal of Marketing, Jg. 44, Nr. 7/8, S. 938-971.

King, C./Grace, D. (2012): Examining the antecedents of positive employee brand-related attitudes and behaviours, in: European Journal of Marketing, Jg. 46, Nr. 3, S. 469-488.

Kirchgeorg, M./Günther, E. (2006): Employer Brands zur Unternehmensprofilierung im Personalmarkt: Eine Analyse der Wahrnehmung von Unternehmensmarken auf der Grundlage einer deutschlandweiten Befragung von High Potentials, Arbeitspapier Nr. 74, Lehrstuhl Marketingmanagement, HHL - Leipzig Graduate School of Management, Leipzig.

Klatetzki, T. (2008): Sozialisation in Gruppen und Organisationen, in: Hurrelmann, K./Grundmann, M./Walper, S. (Hrsg.): Handbuch Sozialisationsforschung, 7. Aufl., Weinheim und Basel, S. 351-371.

Klimecki, R./Gmür, M. (2005): Personalmanagement: Strategien, Erfolgsbeiträge, Entwicklungsperspektiven, 3. Aufl., Stuttgart.

Konczak, L.J./Stelly, D.J./Trusty, M.L. (2000): Defining and measuring empowering leader behaviors: Development of an upward feedback instrument, in: Educational \& Psychological Measurement, Jg. 60, Nr. 2, S. 301-313.

Kotler, P./Armstrong, G.M. (2014): Principles of marketing, 15. Aufl., Boston [et al.].

Kucherov, D./Zavyalova, E. (2012): HRD practices and talent management in the companies with the employer brand, in: European Journal of Training and Development, Jg. 36, Nr. 1, S. 86-104.

Lievens, F. (2007): Employer branding in the Belgian army: The importance of instrumental and symbolic beliefs for potential applicants, and military employees, in: Human Resource Management, Jg. 46, Nr. 1, S. 51-69.

Mahnert, K.F./Torres, A.M. (2007): The brand inside: The factors of failure and success in internal branding, in: Irish Marketing Review, Jg. 19, Nr. 1/2, S. 54-63. 
Maloney, P.B. (2007): Absatzmittlergerichtetes, identitätsbasiertes Markenmanagement, Wiesbaden.

Martin, G./Gollan, P.J./Grigg, K. (2011): Is there a bigger and better future for employer branding? Facing up to innovation, corporate reputations and wicked problems in SHRM, in: The International Journal of Human Resource Management, Jg. 22, Nr. 17, S. 3618-3637.

Meffert, H./Burmann, C. (1996): Identitätsorientierte Markenführung - Grundlagen für das Management von Markenportfolios, in: Meffert, H./Wagner, H./Backhaus, K. (Hrsg.): Arbeitspapiere der Wissenschaftliche Gesellschaft für Marketing und Unternehmensführung e.V., Arbeitspapier Nr. 100, Münster.

Meffert, H./Burmann, C./Kirchgeorg, M. (2012): Marketing, 11. Aufl., Wiesbaden.

Mellor, V. (1999): Delivering brand values through people, in: Strategic Communication Management, Jg. 3, Nr. 2, S. 26-29.

Miles, S.J./Mangold, W.G./Asree, S./Revell, J. (2011): Assessing the employee brand: A census of one company, in: Journal of Managerial Issues, Jg. 23, Nr. 4, S. 491-507.

Mitchell, C. (2002): Selling the brand inside, in: Harvard Business Review, Jg. 80, Nr. 1, S. 99-105.

Morhart, F. (2008): Brand-specific leadership: On its effects and trainability, St. Gallen.

Morhart, F.M./Herzog, W./Tomczak, T. (2009): Brand-specific leadership: Turning employees into brand champions, in: Journal of Marketing, Jg. 73, Nr. 5, S. 122-142.

Morhart, F.M./Jenewein, W./Tomczak, T. (2012): Mit transformationaler Führung das Brand Behavior stärken, in: Tomczak, T./Esch, F.-R./Kernstock, J./Herrmann, A. (Hrsg.): Behavioral Branding: Wie Mitarbeiterverhalten die Marke stärkt, 3. Aufl., Wiesbaden, S. 389-406.

Moroko, L./Uncles, M.D. (2008): Characteristics of successful employer brands, in: Journal of Brand Management, Jg. 16, Nr. 3, S. 160-175.

Mosley, R.W. (2007): Customer experience, organisational culture and the employer brand, in: Journal of Brand Management, Jg. 15, Nr. 2, S. 123-134.

Piebler, R. (2011): Interne Markenführung - Theoretisches Konzept und fallstudienbasierte Evidenz, Wiesbaden.

Punjaisri, K./Evanschitzky, H./Wilson, A. (2009): Internal branding: An enabler of employees' brand-supporting behaviours, in: Journal of Service Management, Jg. 20, Nr. 2, S. 209-226.

Punjaisri, K./Wilson, A. (2007): The role of internal branding in the delivery of employee brand promise, in: Journal of Brand Management, Jg. 15, Nr. 1, S. 57-70.

Punjaisri, K./Wilson, A. (2011): Internal branding process: Key mechanisms, outcomes and moderating factors, in: European Journal of Marketing, Jg. 45, Nr. 9/10, S. 1521-1537.

Punjaisri, K./Wilson, A./Evanschitzky, H. (2008): Exploring the influences of internal branding on employees' brand promise delivery: Implications for strengthening customer-brand relationships, in: Journal of Relationship Marketing, Jg. 7, Nr. 4, S. 407-424.

Ravald, A./Grönroos, C. (1996): The value concept and relationship marketing, in: European Journal of Marketing, Jg. 30, Nr. 2, S. 19-30.

Roj, M. (2013): Die Relevanz der Markenarchitektur für das Employer Branding, Wiesbaden.

Sander, M./Weywara, B. (2006): Markenvertrauen im Rahmen des Markenmanagements, in: Bauer, H.H./Neumann, M.M./Schüle, A. (Hrsg.): Konsumentenvertrauen: Konzepte und Anwendungen für ein nachhaltiges Kundenbindungsmanagement, München, S. 249-264.

Schmidt, H.J./Kilian, K. (2012): Internal Branding, Employer Branding \& Co.: Der Mitarbeiter im Markenfokus, in: transfer Werbeforschung \& Praxis, Jg. 58, Nr. 1, S. 28-33. 
Spannagl, P. (2005): Kundenorientierte Personalentwicklung: Personalmanagement, in: Künzel, H. (Hrsg.): Handbuch Kundenzufriedenheit, Berlin [et al.], S. 73-94.

Sponheuer, B. (2010): Employer Branding als Bestandteil einer ganzheitlichen Markenführung, Wiesbaden.

Strödter, K. (2008): Markencommitment bei Mitarbeitern: Bedeutung der Kongruenz von Mitarbeiter und Marke für das Markencommitment in Unternehmen, Berlin.

Thomson, K./De Chernatony, L./Arganbright, L./Khan, S. (1999): The buy-in benchmark: How staff understanding and commitment impact brand and business performance, in: Journal of Marketing Management, Jg. 15, Nr. 8, S. 819-835.

Tomczak, T./Esch, F.-R./Kernstock, J./Herrmann, A. (2012): Behavioral Branding: Wie Mitarbeiterverhalten die Marke stärkt, 3. Aufl., Wiesbaden.

Vallaster, C./De Chernatony, L. (2005): Internationalisation of services brands: The role of leadership during the internal brand building process, in: Journal of Marketing Management, Jg. 21, Nr. 1/2, S. 181-203.

Vallaster, C./De Chernatony, L. (2006): Internal brand building and structuration: The role of leadership, in: European Journal of Marketing, Jg. 40, Nr. 7/8, S. 761-784.

Van Dyne, L./Graham, J.W./Dienesch, R.M. (1994): Organizational citizenship behavior: Construct redefinition, measurement, and validation, in: Academy of Management Journal, Jg. 37, Nr. 4, S. 765-802.

Van Knippenberg, D./Sleebos, E. (2006): Organizational identification versus organizational commitment: Self-definition, social exchange, and job attitudes, in: Journal of Organizational Behavior, Jg. 27, Nr. 5, S. 571-584.

Van Maanen, J. (1976): Breaking in: Socialization to work, in: Dubin, R. (Hrsg.): Handbook of work, organization, and society, Chicago, Ill., S. 67-130.

Verquer, M.L./Beehr, T.A./Wagner, S.H. (2003): A meta-analysis of relations between person-organization fit and work attitudes, in: Journal of Vocational Behavior, Jg. 63, Nr. 3, S. 473-489.

Wenske, A.V. (2008): Management und Wirkungen von Marke-Kunden-Beziehungen im Konsumgüterbereich - Eine Analyse unter besonderer Berücksichtigung des Beschwerdemanagements und der Markenkommunikation, Wiesbaden.

Wentzel, D./Tomczak, T./Kernstock, J./Brexendorf, T.O./Henkel, S. (2012): Der Funnel als Analyseund Steuerungsinstrument von Brand Behavior, in: Tomczak, T./Esch, F.-R./Kernstock, J./Herrmann, A. (Hrsg.): Behavioral Branding: Wie Mitarbeiterverhalten die Marke stärkt, 3. Aufl., Wiesbaden, S. 81-99.

Wittke-Kothe, C. (2001): Interne Markenführung: Verankerung der Markenidentität im Mitarbeiterverhalten, Wiesbaden.

Zeplin, S. (2006): Innengerichtetes identitätsbasiertes Markenmanagement, Wiesbaden. 
Christoph Burmann, Univ.-Prof. Dr. habil., ist Inhaber des Lehrstuhls für innovatives Markenmanagement (LiM) des Fachbereichs Wirtschaftswissenschaft der Universität Bremen.

Anschrift: Lehrstuhl für innovatives Markenmanagement (LiM), Fachbereich Wirtschaftswissenschaft, Universität Bremen, Hochschulring 4, D-28359 Bremen, Tel.: +49 (0)421/21866572, Fax: +49 (0)421/21866573, E-Mail: burmann@uni-bremen.de

Rico Piehler, Dr., ist Habilitand am Lehrstuhl für innovatives Markenmanagement (LiM) des Fachbereichs Wirtschaftswissenschaft der Universität Bremen.

Anschrift: Lehrstuhl für innovatives Markenmanagement (LiM), Fachbereich Wirtschaftswissenschaft, Universität Bremen, Hochschulring 4, D-28359 Bremen, Tel.: +49 (0)421/21866579, Fax: +49 (0)421/21866573, E-Mail: rico.piehler@uni-bremen.de 